\title{
Perspectives on laparoscopic hysterectomy
}

\author{
Michelle Nisolle
}

Received: 4 January 2010 /Accepted: 4 January 2010 /Published online: 4 February 2010

(C) Springer-Verlag 2010

Keywords Total laparoscopic hysterectomy .

Subtotal hysterectomy complications

Despite the development of numerous hysteroscopic procedures, hysterectomy still represents the most common gynecologic surgery. Nowadays, total or subtotal hysterectomy can be performed laparoscopically as the first total laparoscopic hysterectomy (LH) has been published by H. Reich in 1989 and the first laparoscopic supracervical hysterectomy (LSH) by Donnez and Nisolle in 1993 [1, 2].

Even such procedures are well described in the literature; in USA, hysterectomy is mainly performed abdominally. Indeed, in a series of 538,722 hysterectomies performed for benign disease, the rate of laparoscopic hysterectomy, vaginal hysterectomy, and abdominal hysterectomy were $11.8 \%, 21.8 \%$ and $66.1 \%$, respectively [3].

According to the Cochrande Database review, it is, however, recommended to avoid abdominal hysterectomy as often as possible in order to favor vaginal hysterectomy [4].

In a recent prospective randomized study comparing vaginal hysterectomy $(\mathrm{VH})$, laparoscopic-assisted vaginal hysterectomy (LAVH), and total laparoscopic hysterectomy in women with benign uterine disease, Drahonovsky et al. also concluded that $\mathrm{VH}$ and LAVH should be the preferred techniques. For the authors, only experienced laparoscopists should perform LH and in cases where VH and LAVH are quite impossible [5].

How can we explain that laparoscopic hysterectomy is not worldwide performed?

Is it due to a long learning curve or to a high rate of complications associated to the procedure?

\section{Nisolle $(\square)$}

Department of Obstetrics and Gynecology, University of Liège,

CHR de La Citadelle, Boulevard du Douzième De Ligne, 1,

4000 Liège, Belgium

e-mail: michelle.nisolle@chu.ulg.ac.be
In a recent multicenter retrospective cohort study, van Evert et al. evaluated the peri- and postoperative outcomes in women undergoing either LSH or LH. They clearly demonstrated that the rate of complications (short- and long-term) was similar in both techniques: $17 \%$ in LSH and $15 \%$ in LH [6], but differences were observed when they compared short-term and long-term complications. Indeed a higher rate of short-term complications was noted in LH group than in LSH group (12\% vs $3 \%$ ). However in LSH group, the long-term complications rate was found to be higher when compared to that observed in LH group (15\% vs 3\%).

The overall rate of complications observed in their series ( $17 \%$ in LSH and $15 \%$ in LH) is similar to that observed in previously published series [7].

In previous studies, the rate of major complications has been estimated to be $4 \%$.

Whatever the technique used, the aim of gynecologists performing laparoscopic procedures is to reduce the rate of complications at short or long term.

In order to decrease the rate of long-term complications associated to LSH, gynecologists have to select patients for this type of procedure. For example, in patients with pelvic organ prolapse, undergoing laparoscopic sacrocolpopexy associated with hysterectomy, LSH should be preferred in order to reduce the postoperative risk of mesh erosion. On the contrary, in cases of cervical pathology or deep infiltrating endometriosis involving the cervix, LSH should be avoided in order to decrease the risk of reoperation due to cervical stump problems or persistent dyspareunia [8].

One advantage of LSH is the absence of vaginal opening and therefore the absence of contamination of peritoneal cavity by vaginal bacteria and the potential risk of postoperative peritonitis. In the study of van Evert et al., 


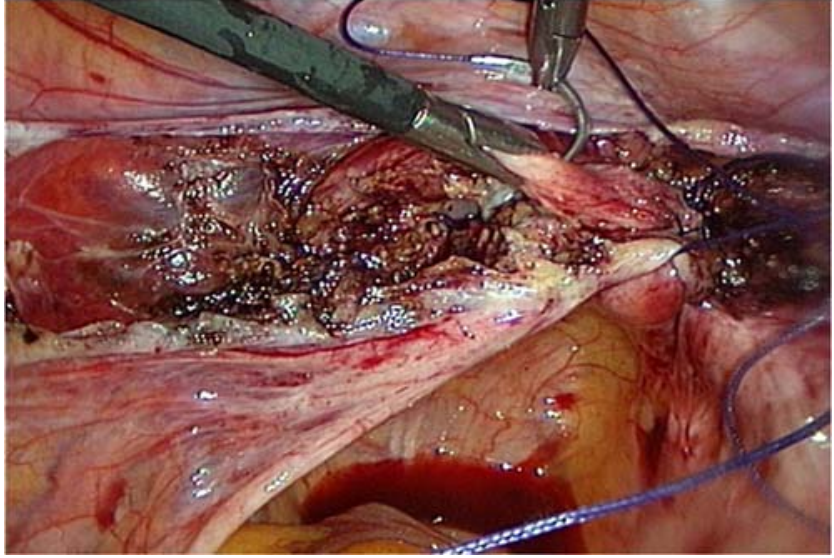

Fig. 1 Laparoscopic vaginal vault closure

vaginal vault hematoma was observed in the LH group, and the authors evocated that the vaginal vault closure achieved vaginally could be the cause of hematoma infection. The advantage of laparoscopic closure of the vaginal vault is the perfect control of hemostasis at the end of the procedure and the absence of vaginal manipulation which also could favor migration of vaginal bacteria to the pelvis (Fig. 1).

Though in cases of LSH, morcellation of the specimen is required, and precautions must be taken to avoid any complication or loose of large uterine specimens in the abdominal cavity.

In order to decrease the rate of short-term complications associated to LH, adequate laparoscopic skills are needed, as well as knowledge of the procedure and use of specific endoscopic material developed for facilitating laparoscopic surgery such as uterine manipulator, morcellator, endoloop, etc.

Uterine manipulation can be considered as a limiting factor for LH, especially in cases of enlarged uterus. According to the comparative survey of various uterine

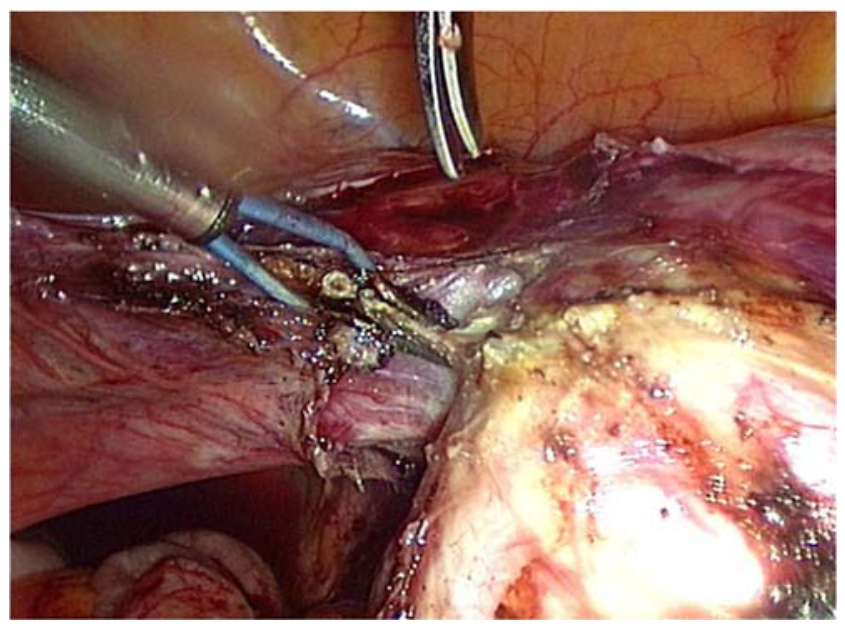

Fig. 2 Uterine manipulation and visualization of left uterine artery

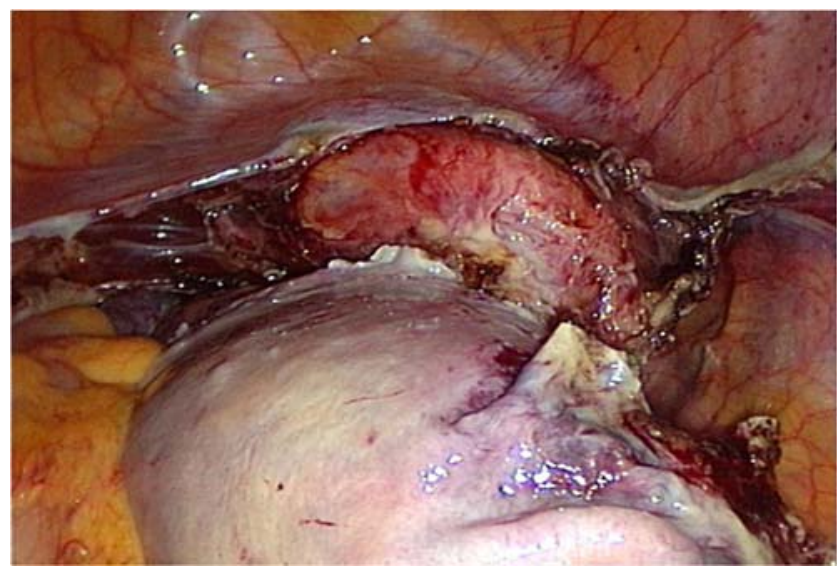

Fig. 3 Vaginal fornices presentation

manipulators published by Mettler and Nikam, the criteria of efficacious manipulators are the following [9]:

- To mobilize the uterus in several directions (Fig. 2)

- To present the vaginal fornices (Fig. 3)

- To maintain the pneumoperitoneum (Fig. 4)

The use of linear cutter stapling device for securing uterine vascular pedicles has been suggested during the achievement of laparoscopic hysterectomy [10]. According to the retrospective analysis of their series of 1,275 laparoscopic hysterectomies, the authors concluded that the risk of ureteral injury is not increased when compared to abdominal hysterectomy and to the use of bipolar diathermy.

Obesity is sometimes a contraindication for laparoscopic hysterectomy, but very recently, Chopin et al. demonstrated that in obese and overweight patients, LH was not associated with increased risk of complications but only with increased operating time [11].

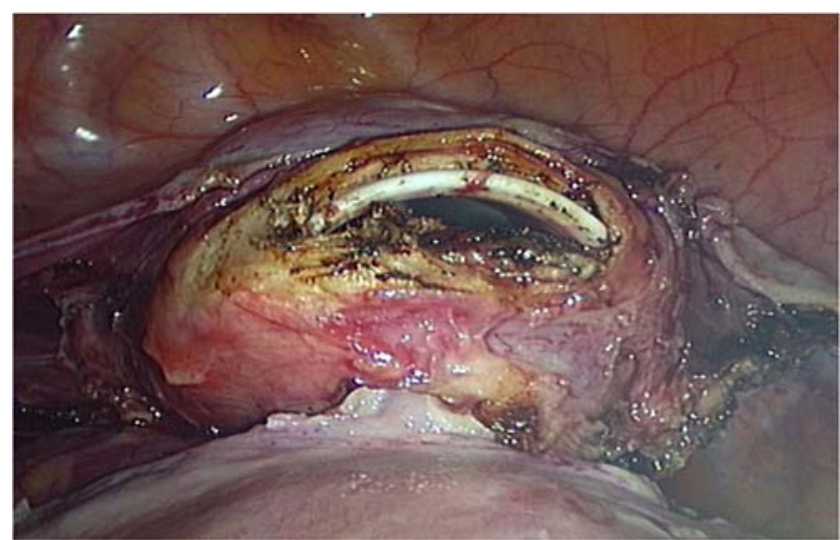

Fig. 4 Maintenance of the pneumoperitoneum with the uterine manipulator 
In summary, limiting factors of LH associated to the patient's conditions are essentially fixed enlarged uterus due to previous surgery or advanced stage of endometriosis.

The surgeon's inexperience is a real limiting factor for $\mathrm{LH}$, and continuing the endoscopic learning by using laparoscopic skills stimulator and by participating to training courses and life surgery course is strongly suggested to gynecologists [12].

In conclusion, gynecologists performing laparoscopic hysterectomy should be aware of the following:

- Subtotal hysterectomy is an easier procedure than $\mathrm{LH}$ as no dissection of the cervix is required neither the vaginal closure, but uterine morcellation is needed, and precautions must be taken to avoid damage to pelvic organs.

- Subtotal hysterectomy for benign disease should be proposed to carefully selected patients (without cervical anomalies and deep infiltrating endometriosis).

- The absence of vaginal opening should reduce the risk of hematoma and peritonitis.

- Total laparoscopic hysterectomy is feasible in the majority of cases, but experience is needed as well as adapted endoscopic material.

- Laparoscopic vaginal vault closure could avoid the risk of vaginal vault hematoma as visual control of any bleeding at the time of vaginal closure is ideally obtained. The absence of vaginal manipulation during the laparoscopic suture could also theoretically decrease the rate of postoperative peritonitis.

- The goal of the gynecologists is to propose surgical procedure associated with a low rate of complications at short or long term.

- A correct selection of patients and an appropriate learning curve should decrease not only the risks of per-operative complication but also the risk of major complications.

- In the future, if studies revealed that hysterectomy performed by robotics is associated with a short learning curve and with a low rate of complications, it could be offered to many patients.

\section{References}

1. Reich H, De Caprio J, Mc Glynn F (1989) Laparoscopic hysterectomy. J Gynecol Surg 5:213-216

2. Donnez J, Nisolle M (1993) Laparoscopic supracervical (subtotal) hysterectomy (LASH). J Gynecol Surg 9:91-94

3. Wu JM, Wechter ME, Geller EJ, Nguyen TV, Visco AG (2007) Hysterectomy rates in the United States, 2003. Obstet Gynecol 110:1091-1095

4. Nieboer TE, Johnson N, Lethaby A, Tavender E, Curr E, Garry R, van Voorst S, Mol BW, Kluivers KB (2009) Surgical approach to hysterectomy for benign gynaecological disease. Cochrane Database Syst Rev 8(3):CD003677

5. Drahonovsky J, Haakova L, Otcenasek M, Krofta L, Kucera E, Feyereisl J (2009) A prospective randomized comparison of vaginal hysterectomy, laparoscopic assisted vaginal hysterectomy, and total laparoscopic hysterectomy in women with benign uterine disease. Europ J Obstet Gynecol Reprod Biol (in press)

6. van Evert JS, Smeenk JM, Dijkhuizen FP, de Kruif JH, Kluivers KB (2009) Laparoscopic subtotal hysterectomy versus laparoscopic total hysterectomy: a decade of experience. Gynecol Surg. doi:10.1007/ s10397-009-0529-8

7. Chapron C, Laforest L, Ansquer Y, Fauconnier A, Fernendez B, Breart G, Dubuisson JB (1999) Hysterectomy techniques used for benign pathologies: results of a French multicentre study. Hum Reprod 14:2464-2470

8. Nezhat CH, Nezhat F, Roemisch M, Seidman DS, Nezhat C (1996) Laparoscopic trachelectomy for persistent pain and endometriosis after supracervical hysterectomy. Fertil Steril 66:925-928

9. Mettler L, Nikam YA (2006) A comparative survey of various uterine manipulators used in operative laparoscopy. Gynecol Surg 3(4):239 243

10. McMaster-Fay RA, Jones RA (2006) Laparoscopic hysterectomy and ureteric injury: a comparison of the initial 275 cases and the last 1,000 cases using staples. Gynecol Surg 3(2):118-21

11. Chopin N, Malaret JM, Lafay-Pillet MC, Fosto A, Foulot H, Chapron C (2009) Total laparoscopic hysterectomy for benign uterine pathologies: obesity does not increase the risk of complications. Hum Reprod 24(12):3057-3062

12. Kolkman W, van de Put MA, Wolterbeek R, Trimbos JB, Jansen FW (2008) Laparoscopic skills stimulator: construct validity and establishment of performance standards for residency training. Gynecol Surg 5:109-114 